\title{
Low Power Field
}

National Cancer Institute

\section{Source}

National Cancer Institute. Low Power Field. NCI Thesaurus. Code C67307.

The area visible in one view field of the microscope under the low magnification of the objective being used in microscopy. 